\title{
Potencial da aprendizagem baseada-em-jogos: Um caso de estudo na Universidade do Algarve
}

\author{
Tatiana Kikot ${ }^{1}$, Silvia Fernandes ${ }^{1}$, Gonçalo Costa $^{2}$ \\ ruskikot87@gmail.com, sfernan@ualg.pt, goncalo.j.m.costa@gmail.com \\ ${ }^{1}$ Faculdade de Economia, Universidade do Algarve, Campus Gambelas, 8005-139 Faro, Portugal \\ ${ }^{2}$ Centre for Computing and Social Responsibility, De Montfort University, Leicester, UK
}

DOI: 10.17013/risti.16.17-29

\begin{abstract}
Resumo: Casos e desafios atuais incluem novos modos de aprendizagem e desenvolvimento de competências. Cada vez é mais necessário investir em si mesmo a nível de habilidades e criatividade. Os jogos e simulações, conjugados eventualmente com ambientes virtuais, representam um potencial para novas carreiras ou adaptações. Um caso é o do jogo Cesim SimBrand que envolve os estudantes num ambiente de marketing virtual onde obtêm incomparável experiência em trabalho de equipa e resolução de problemas. A maioria dos estudantes inquiridos destacou o resultado de maior envolvimento na aprendizagem associado à dinâmica realista do jogo. Tais jogos podem ser complementados com mundos virtuais ou realidade aumentada no sentido de analisar o enquadramento com outras atividades/ negócios ou em outros contextos. Isso terá implicações a considerar nos programas, modos de ensino/aprendizagem, locais de ensino e potenciais carreiras.
\end{abstract}

Palavras-chave: jogos, simulações, ensino superior, aprendizagem, realidade aumentada.

\section{Potential of game-based learning: A case study at the University of Algarve}

\begin{abstract}
Current cases and challenges include new ways of learning and skills' development. It is increasingly necessary to invest on ourselves in terms of competences and creativity. Games and simulations, possibly associated with virtual environments, represent a potential for new careers or adaptations. A case is the game Cesim Simbrand which involves students in a virtual marketing environment where they get unmatched experience on teamwork and problem solving. Most students surveyed referred the result of greater involvement in learning associated with the realistic dynamics of the game. Such games can be complemented with virtual worlds and augmented reality in order to analyze the integration in other activities/ businesses or other contexts. This will have implications to consider in programs, modes of teaching/learning, learning locations and potential careers.
\end{abstract}

Keywords: games, simulations, higher education, learning, augmented reality. 


\section{Introdução}

Atualmente, face à instabilidade resultante de novas dinâmicas socio-económicas, as pessoas e organizações devem readaptar-se para persistirem num ambiente em constante mudança. Alguns dos atuais desafios incluem novos modos de aprendizagem, trabalho e desenvolvimento. Em muitos contextos, a fim de ter vida profissional, é necessário investir em si mesmo a nível de habilidades, competências e criatividade. Construir contactos/redes e assumir riscos para descobrir e implementar novos conhecimentos e técnicas podem dar origem a novas carreiras (Hoffman \& Casnocha, 2013; Aldrich, 2009).

Das tecnologias de informação e comunicação (TIC) têm resultado suportes interessantes na educação, empresas e sociedade em geral (Castells, 2009). Alguns resultados incluem dispositivos móveis (smartphones, tablets) que podem aumentar o envolvimento dos alunos em diversas atividades com aplicações inovadoras, redes sociais e outras ferramentas web 2.o (blogues, wikis, etc.) que podem dar aos alunos um papel mais ativo na sua educação e conteúdos (tornando-se prosumers - produtores e consumidores (Martin et al., 2011).

Segundo especialistas e estudos relacionados (Horizon Report, 2014) as tecnologias móveis, ubíquas (sensíveis ao contexto), de simulação (jogos) e realidade aumentada tendem a criar vários contextos de educação e trabalho futuros (Martin et al., 2011). Os jogos digitais podem apoiar a educação (Kardynal, 2009), uma vez que simulações representam modelos dinâmicos de situações reais. Essa reconstrução de realidades permite que os 'jogadores' (alunos, utilizadores) antecipem consequências de suas decisões e potencial repercussão no sistema socio-económico exterior (McClarty, 2012). Outra vantagem é a possibilidade de registar e avaliar as sucessivas experiências durante a simulação, o que promove novo conhecimento acerca do mundo real (Siewiorek et al., 2012). Trata-se de uma solução interessante para envolver e preparar os alunos em compreender melhor os contextos exteriores (Findling, 2008). As habilidades construídas através de simulações e jogos sérios (serious games) tendem a desafiar universidades e institutos a melhorar seus programas, conteúdos e atividades (Aldrich, 2009).

Os alunos, ao participarem em atividades online também deixam um 'rasto' (track) de dados que podem ser objeto de mineração (data mining) para análise de perfis e reações. É importante compreender como é que um jogo ou simulação pode ser uma solução para preparar melhor os estudantes para o atual ambiente económico em mutação (Findling, 2008). Este artigo pretende expôr tendências e resultados em termos de perceções e experiências de alunos e docentes com jogos de gestão no ensino superior, como é o caso do Cesim SimBrand na Universidade do Algarve. Para tal, tem a seguinte estrutura: após uma breve introdução ao tema, segue-se uma descrição das tendências em termos de jogos sérios e simulações e suas implicações no ensino/ aprendizagem. Na terceira secção apresentam-se alguns resultados da aplicação de um jogo de gestão no ensino superior, comparando a perspetiva do docente com a dos alunos. A quarta secção reflete sobre desafios da realidade aumentada e sua potencial integração com jogos/simulações no ensino. E a quinta secção descreve as principais conclusões do presente trabalho. 


\section{Aprendizagem baseada-em-jogos}

Uma mudança está a emergir na área de prática pedagógica em campus universitários, de como estudantes numa ampla variedade de disciplinas podem 'aprender criando' em vez de só consumirem conteúdos (Kikot et al., 2013). Nos últimos anos, denota-se um crescimento de comunidades de geração/partilha de conhecimento e de projetos crowdfunded como meios de aprendizagem ativa. Cursos e planos de formação de várias disciplinas estão num processo de mudança de modo a enquadrar o uso e criação de media, o design e empreendedorismo. Bibliotecas de campus cada vez mais disponibilizam plataformas que suportam a produção e criatividade (Horizon Report, 2014). Por outro lado, a literatura (Yang e Wu, 2012) mostra que jogos ou simulações podem ser meios eficazes de adquirir aptidões para compreender melhor os contextos reais em relação aos ambientes tradicionais ou até híbridos (online junto com presencial - blended learning). A crescente complexidade do ambiente atual é uma oportunidade e, simultaneamente, um desafio para o recurso a simulações e jogos específicos (Golding, 2009). Pois um jogo de simulação permite uma recriação digital de algo real podendo ter características como: concorrência, regras, ganhar, perder, etc.

Existem vários tipos de jogos: digitais comerciais, principalmente orientados para entretenimento/lazer; de aprendizagem (GBL); e jogos sérios (serious games - tipo mais abrangente que o anterior) que permitem a aprendizagem académica e pré-profissional (Connolly et al., 2012). Os termos serious games (SG) ou game-based learning (GBL) ou jogos de simulação são por vezes usados como sinónimos (Corti, 2009), apesar dos SG terem efeitos mais amplos na formação e comportamento em negócios, indústria, saúde e educação. A principal distinção reside na intenção do utilizador e no grau de proximidade com a realidade: se o objetivo principal é a aprendizagem pode ser classificado de jogo sério; se incide num treinamento o jogo pode ser considerado como simulação (Johnston \& Whitehead, 2006). Ao contrário dos jogos de vídeo educativos (tradicionalmente dirigidos ao sistema de ensino primário e secundário), os jogos sérios são concebidos para uma grande variedade de públicos que requerem habilidades de pensamento com nível avançado.

Em termos de simulações, existem as de categoria analítica e as de instrução (Aldrich, 2009). As primeiras procuram replicar um determinado fenómeno para que o utilizador o analise no sentido de apoiar a tomada de decisão; as outras são utilizadas para objetivos de formação. O potencial dos jogos/simulações no ensinoaprendizagem, aliado ao ambiente online, sugere uma mudança nos paradigmas de educação em preparar os alunos/utilizadores em competências independentes do meio académico. Pela colaboração e experimentação que potenciam, criam competências que combinam o melhor dos dois ambientes (como defende a Fundação Francisco Manuel Santos em várias projetos e conferências sobre esta temática $\left.{ }^{1}\right)$. O próprio uso de jogos portáteis entre os jovens torna a aprendizagem móvel, o que é relevante porque assim podem até usar os momentos livres para aprendizagem (Virvou \& Alepis, 2005). Outros estudos realçam a aprendizagem baseada-em-jogos como meio de melhorar o

${ }^{1}$ Como por exemplo a Conferência intitulada “Admirável Mundo Novo" (Junho, 2015) http://www.ffms.pt/conferencia-depois/1050/admiravel-mundo-novo\#inscricoes. 
raciocínio (Eastwood \& Sadler, 2013) ou o envolvimento dos estudantes (Anderson, 2006), embora alguns discutam o grau de aprendizagem real (Guillén-Nieto \& AlesonCarbonell, 2012). Algumas razões têm a ver com: 1) o conhecimento teórico obtido em aula não fornecer uma visão cabal dos aspetos de negócio; e 2) as explicações dos docentes muitas vezes não incluirem experiências práticas e desenvolvimento de habilidades fundamentais numa futura carreira (Sorensen, 2011).

\section{Um caso de aplicação no ensino superior}

Antes de apresentar alguns resultados da adesão a jogos de suporte à aprendizagem no ensino superior em Portugal, seguem-se alguns gráficos com estatísticas europeias ${ }^{2}$ sobre emprego em atividades intensivas em conhecimento, e níveis de qualificação educativa e de governação eletrónica $(e-g o v)$. Um objetivo é refletir na relação da situação de Portugal nestas 3 áreas e o seu grau de adesão e difusão de jogos/simulações de aprendizagem no ensino e gestão.

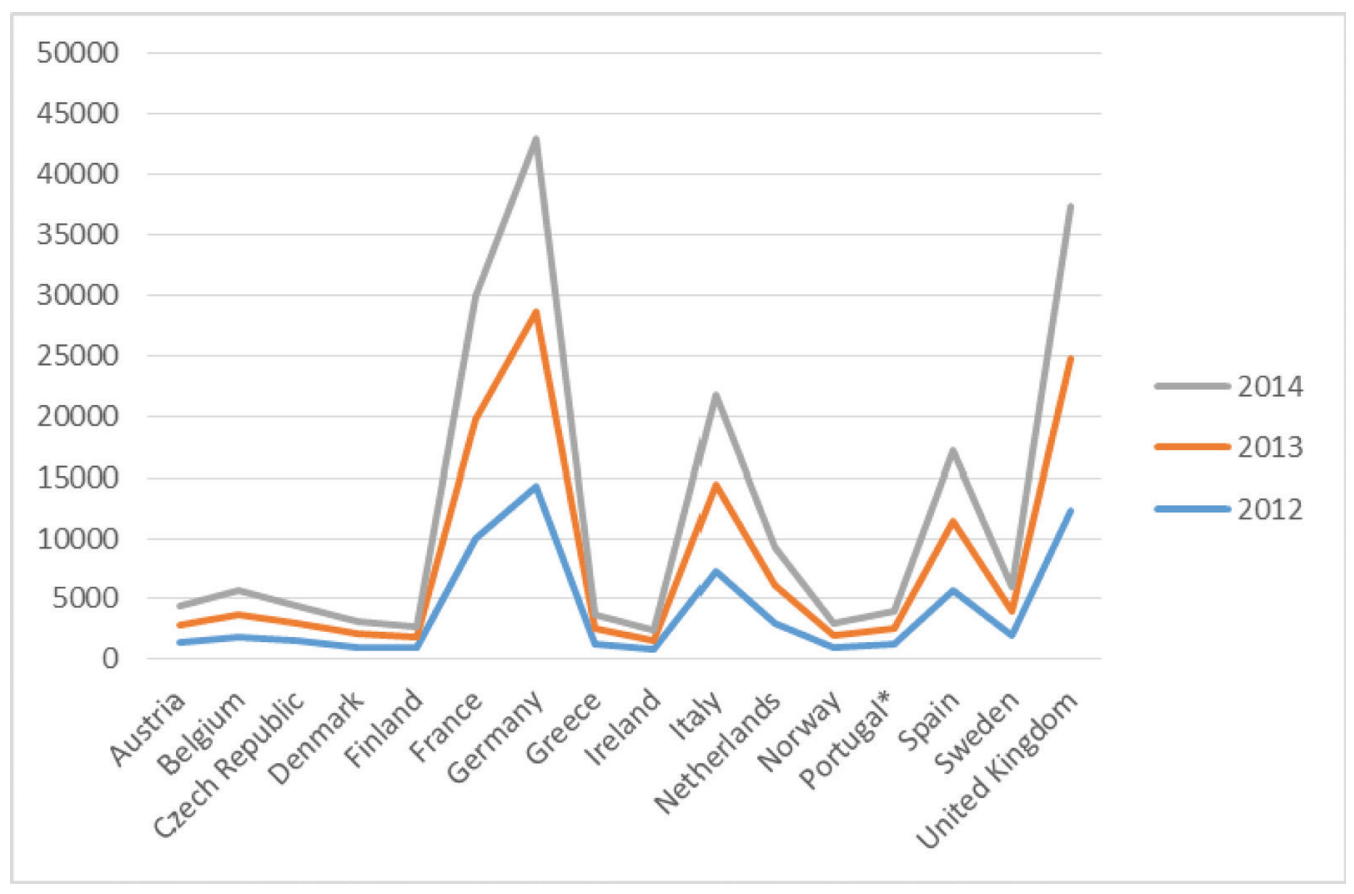

Figura 1 - Emprego em atividades intensivas em conhecimento

2 Com base em dados do Eurostat- http://ec.europa.eu/eurostat. 


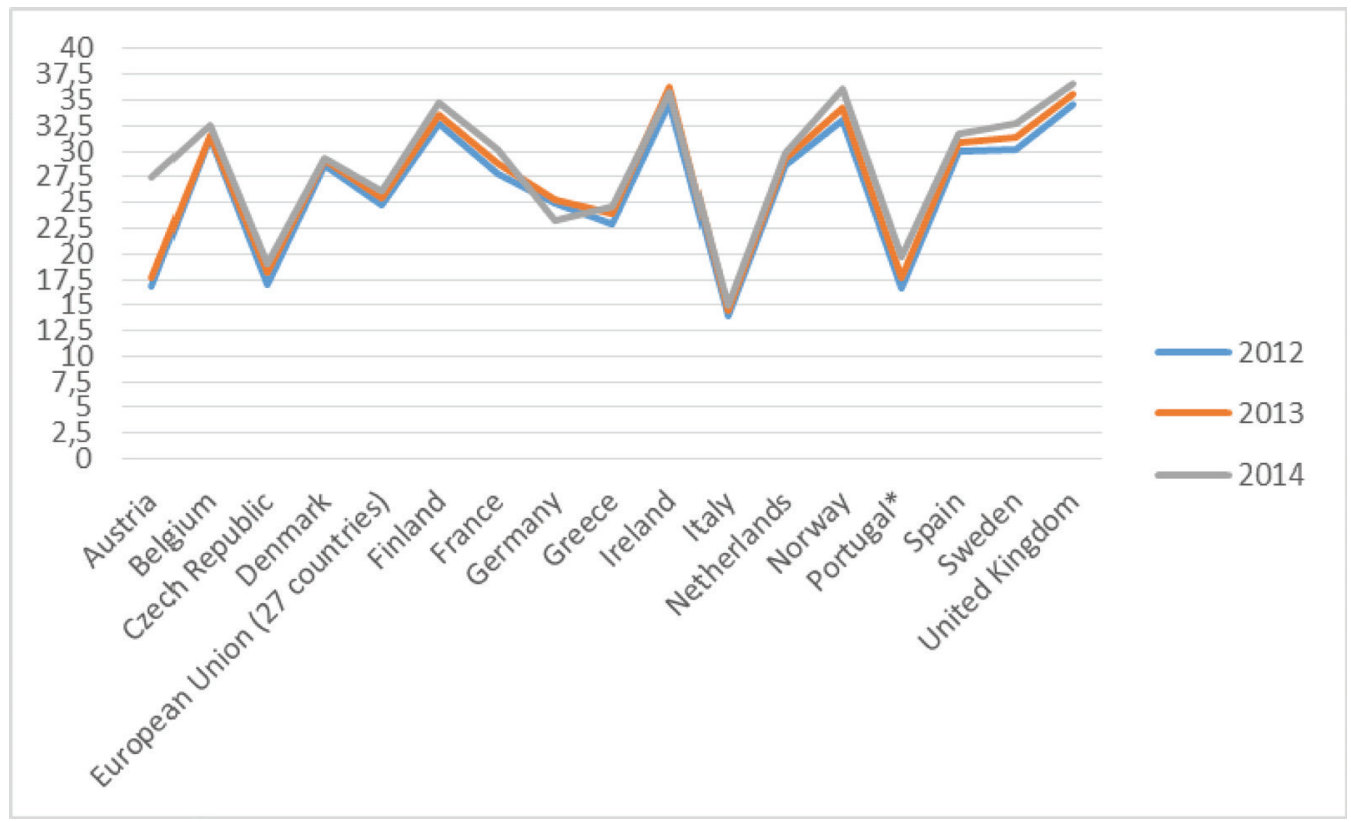

Figura 2 - Grau de qualificação educativa

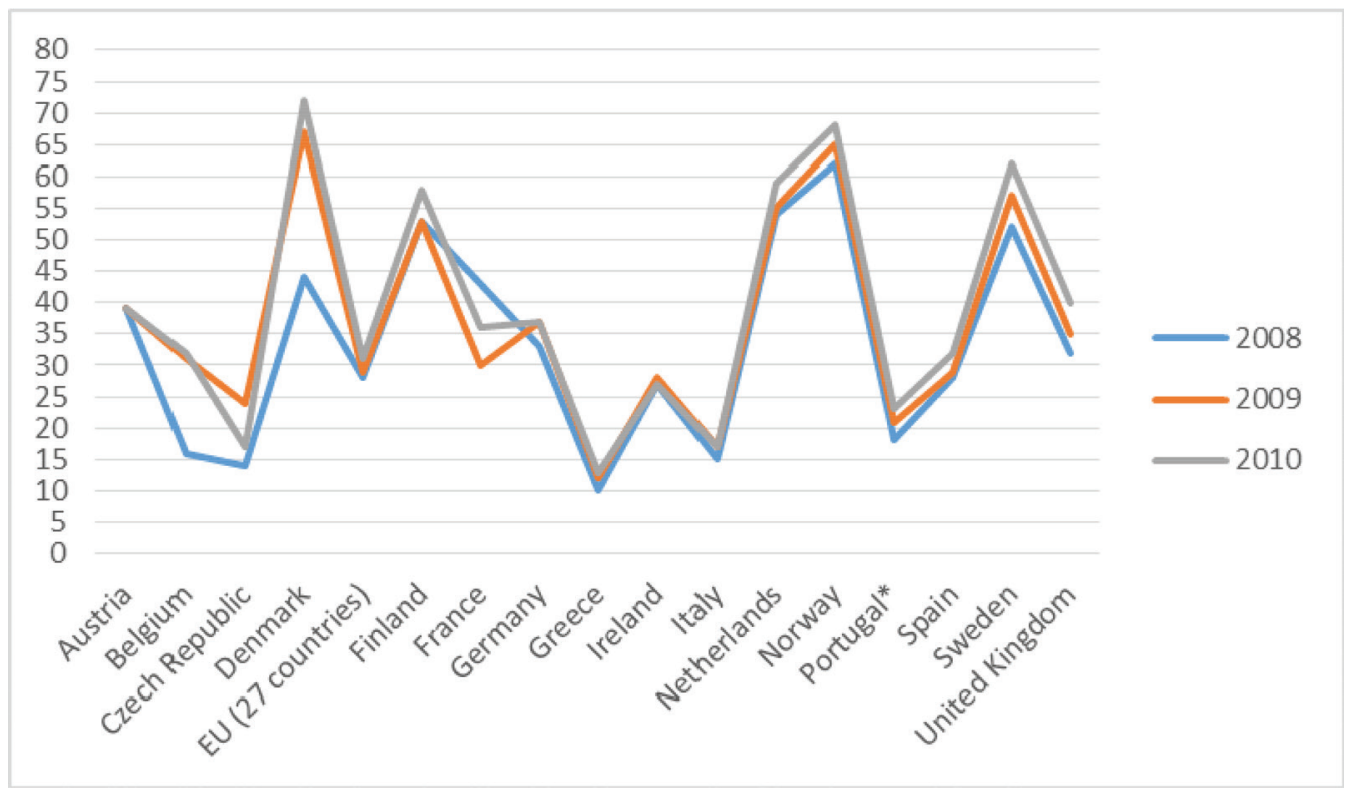

Figura 3 - Grau de uso da governação eletrónica (e-gov) 
Nestas 3 áreas (Figuras 1 a 3) Portugal revela níveis abaixo da média europeia, embora venha experimentando um aumento dos mesmos no tempo. Uma menor expressão da aprendizagem baseada-em-jogos no ensino e gestão em Portugal pode estar relacionada com os baixos níveis revelados nestas áreas, comparativamente a outros países (Lifelong Learning Programme, 2012). Por exemplo, uma maior inovação e suporte tecnológico no ensino têm permitido novos perfis técnicos e de conhecimento que contribuem para aumentar o nível de atividades baseadas em conhecimento e em governação eletrónica (CMR, 2014). Apesar do potencial dos jogos e simulações no ensino-aprendizagem, várias barreiras foram identificadas em termos da sua adoção e difusão nos contextos educativos (Eastwood \& Sadler, 2013). Estudos recentes sobre jogos digitais no seio académico constatam aspetos contraditórios quanto à eficácia do envolvimento dos estudantes (Yang \& Wu, 2012; Papastergiou, 2009). No entanto, várias universidades portuguesas têm aderido à aprendizagem baseada-em-jogos por criar um modo de lecionar que vai ao encontro do uso crescente de tecnologias pelos alunos. A Universidade do Algarve tem incluido recentemente jogos de simulação em disciplinas opcionais de alguns cursos, especialmente relacionados com marketing e gestão. Os estudantes têm obtido alguns benefícios de aprendizagem tais como: 1) exploram cenários económicos complexos sem incorrer em penalizações financeiras/fiscais; 2) estão mais envolvidos com os assuntos e os docentes aumentando sua participação e motivação (Salas et al., 2009); 3) desenvolvem habilidades estratégicas, de decisão e comportamentais; e 4) implementam decisões reais úteis (Wall \& Vian, 2008; Zantow \& Knowlton, 2005).

\subsection{O Caso do Cesim SimBrand}

O jogo Cesim SimBrand envolve os estudantes num ambiente de marketing virtual, no contexto de uma empresa de telemóveis, onde competem com outras equipas de modo a obter o escalão mais alto (que torna a empresa bem sucedida). Esta plataforma requer a atenção dos participantes em gerir um portfólio de produtos adequando aspetos qualitativos e quantitativos aos segmentos alvo (preços, promoção e canais adequados às preferências dos segmentos) e gerir as decisões posteriores de desenvolvimento e vendas. Os participantes ficam assim a compreender as diferentes partes do processo de decisão de marketing, da sua relação com as outras equipas e do seu impacto nos resultados da empresa. Os estudantes obtêm incomparável experiência em trabalho de equipa e resolução de problemas (Cesim, 2013).

\subsubsection{Abordagem de investigação}

Foi efetuado um estudo sobre este jogo num curso da Universidade do Algarve, em que os dados recolhidos resultaram de um questionário com perguntas de tipo fechado e aberto, sendo este último útil na recolha de opiniões (Burns, 2000). O questionário foi elaborado através do aplicativo LimeService ${ }^{3}$, sendo enviado à turma objeto de estudo um e-mail descritivo com o link para o mesmo. A posterior análise dos dados baseou-se: 1) numa abordagem quantitativa para as questões fechadas (Alaranta, 2006); e 2) numa abordagem hermenêutica para as questões abertas, que relaciona as partes com o todo (Geanellos, 2000). Esta última abordagem é relevante na determinação do significado

3 Acessível em: https://www.limeservice.com. 
de certos dados textuais (Radnitzky, 1970). O questionário dirigido aos estudantes teve a seguinte estrutura:

- $\quad$ secção 1 (perfil do participante, 8 questões): informação biográfica como por exemplo género, idade, experiência de trabalho, experiência anterior em jogos sérios ou simuladores e frequência de uso desse tipo de jogos;

- $\quad$ secção 2 (aprendizagem baseada-em-jogos, 8 questões): inclui sensibilidade à GBL, bem como identificar as principais características da mesma e papel do docente durante o jogo;

- secção 3 (caracterização do jogo Cesim SimBrand, 4 questões): experiência dos estudantes com jogos sérios e de simulação, suas perceções de utilidade e vontade de experimentar esses jogos em outras disciplinas;

- secção 4 (qualificações, 4 questões): nível de preparação dos estudantes para jogar o Cesim SimBrand e satisfação de suas expectativas.

O jogo Cesim SimBrand é usado na disciplina de 'Simulação de Marketing' (do curso de Licenciatura em Gestão) para melhorar as habilidades e conhecimento dos alunos na área de marketing. Durante o ano letivo de 2014/15, cerca de 32 estudantes estavam inscritos. Os estudantes jogam durante 10 fases (uma fase corresponde a um ano da empresa), fora da aula, pois discutem depois em aula os seus resultados e decisões (e as diferenças). O principal atributo deste jogo consiste na sua ligação a várias matérias do programa do curso em que se insere, a fim de reforçar conceitos e melhorar a partilha de conhecimentos. Após cada fase, o docente distribui um quiz em aula que incentiva os estudantes à compreensão de suas decisões. Por exemplo, enquanto os estudantes escolhem o modo de publicidade, canais de comunicação e investimento, o docente pede para descreverem procedimentos e estimarem os custos por produto. Nesta discussão, o docente relaciona assim conceitos com os procedimentos no seio do simulador.

Após 2 fases de jogo, estabeleceram-se conversas informais de modo a apreender as convicções e expetativas dos participantes quanto ao uso do jogo. Eles expressaram grande interesse e entusiasmo por este novo meio (ou ferramenta) de ensino, referindo envolvimento ativo e apoio à aprendizagem como características-chave. Este entusiasmo e confiança podem ser justificados pelo ambiente do jogo ser similar ao de uma empresa real.

\subsubsection{Principais resultados e trabalho futuro}

Dos questionários enviados aos estudantes, cerca de metade estavam completos. A maioria dos respondentes tem entre 21 e 25 anos de idade e é do género masculino (secção 1 do inquérito). Das restantes questões do inquérito, as que mais realçamos são as diretamente relacionadas com o uso de jogos por parte dos respondentes, duração, tipos, experiência anterior com jogos sérios e experiência com o jogo em causa em termos de expetativas, performance, trabalho de equipa, resultados e dificuldades (secções 3 e 4 do inquérito).

Quando questionados sobre o uso de jogos, a resposta comum foi sim; contudo a maioria dos participantes (46\%) afirmaram que o fazem durante menos de 1 hora por dia. Cerca de $33 \%$ responderam entre 1 a 2 horas, e apenas $13 \%$ de 2 a 3 horas por dia. Quanto ao tipo, os jogos de aventura são os mais populares seguidos de simulações nas áreas do desporto ou aviação. Nenhum participante teve experiência anterior com 
jogos/simulações na área da educação, embora a maioria (58\%) referisse a importância da aprendizagem baseada-em-jogos. Sobre as vantagens destes jogos, 90\% referiram um maior envolvimento na aprendizagem e uma forma de apresentação ilustrativa por permitirem uma competição realista. Esta foi uma característica-chave para os estudantes pois permite-lhes adquirir um melhor entendimento das principais áreas de negócio.

A característica trabalho de equipa é controversa pois alguns respondentes apontam-na como vantagem (expansão da decisão) enquanto outros ficaram insatisfeitos com a sua performance em resultado da falta de responsabilidade ou capacidade de negociação dos seus colegas. Algumas dificuldades foram apontadas na descrição das regras do jogo e na distribuição de resultados. O papel do docenteincidiu na orientação do processo e motivação (mas o presente trabalho não foca a perspetiva do docente). A principal preocupação dos estudantes foi a performance de jogo, isto é, o nível de realidade e diversidade dos cenários de decisão. Um aspeto a realçar foi o nível de autonomia, em que os alunos preferiram em geral tomar as suas decisões em aula. Foram referidas outras vantagens como: mudanças nos procedimentos de trabalho (sobretudo novos cenários de negócio), experiências "reais" e compreensão dos tópicos da matéria. Adicionalmente, quanto ao seu grau de preparação, 53\% dos participantes sentiram-se preparados especialmente após as duas primeiras fases do jogo.

No final do processo, os estudantes sentiram-se satisfeitos pois consideram que aprenderam mais do que inicialmente previam. A experiência geral foi positiva levando os estudantes a expressar a sua vontade de explorar a aprendizagem baseada-em-jogos (GBL) em outras disciplinas, tais como 'Estratégia' ou 'Análise de negócio'. No futuro, o questionário será complementado com entrevistas a grupos-foco (focus groups) pois, enquanto os questionários individuais permitem compreender as pessoas ou opiniões, os grupos-foco permitem a expressão de sentimentos ou perceções não expressas individualmente (Gall et al., 2003). Esta opção pode ter particular utilidade para aspetos sensíveis das opiniões dos estudantes (como por exemplo: papel do docente durante o jogo, porque o trabalho em equipa pode ser controverso, etc.).

Por outro lado, existe um desafio a ter em conta que são as aplicações de realidade aumentada e sua potencial integração ou complementaridade com os jogos/ simulações de aprendizagem para um ensino diversificado, flexível e inovador.

\section{Desafios da realidade aumentada na aprendizagem}

Novas tendências em tecnologia e mudanças no software e infraestruturas na internet, bem como na cultura de participação em ambientes virtuais, alteram não só o modo como as pessoas procuram informação sobre produtos/serviços mas também como os usam (Sizo et al., 2010). Existe um desafio crescente de formação pela internet, desde as plataformas de e-learning até entrar em mundos virtuais como participantes ativos

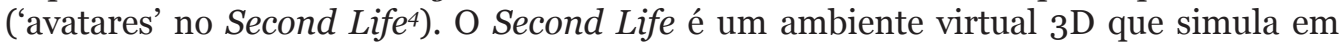
muitos aspetos a vida real da sociedade. Dependendo do tipo de uso pode ser encarado como um jogo, ou simulador, ou comércio virtual ou rede social. Dada a animação 3D,

4 Em http://www.secondlife.com. 
os 'avatares' podem experimentar interações como simulação para decisões ainda mais reais (contextualizadas no exterior). Os jogos de gestão e aprendizagem poderão ser complementados com estas plataformas no sentido dos próprios participantes testarem o enquadramento com outros colegas, cursos, atividades e negócios até em outros países, culturas ou contextos (Figura 4). Trata-se de um potencial ainda maior de simulação e aproximação à realidade e consequente antecipação de soluções futuras mais assertivas (Ixmatlahua et al., 2015).

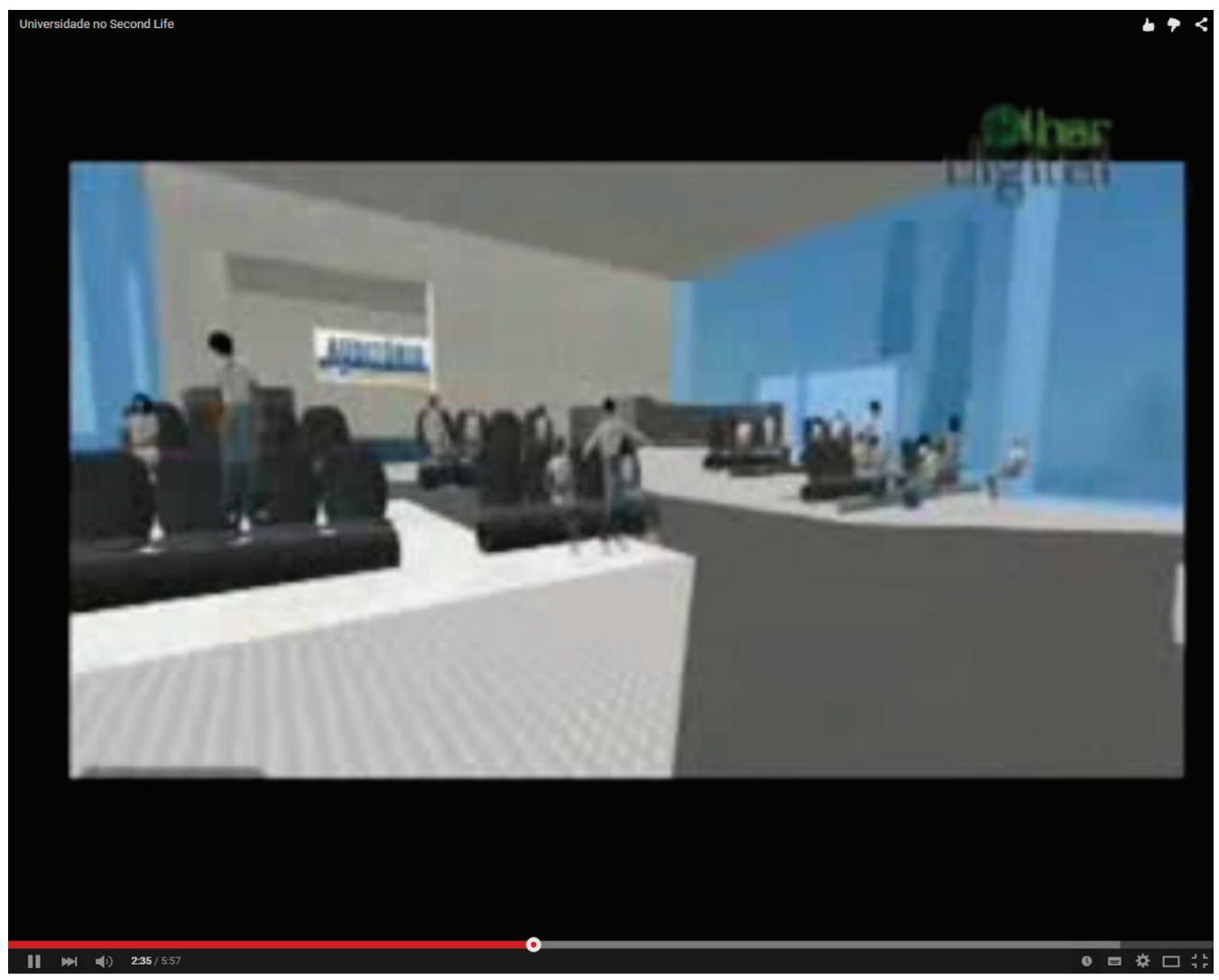

Figura 4 - Salas de aula, conferência ou negócio no Second Life

O Second Life inclui dezenas de cursos, matrículas, espaços; experiências interativas em $2^{\mathrm{a}}$ vida pois os participantes podem ganhar dinheiro virtual; entre outras realidades (comunidades). Até públicos diferentes poderão ser captados para cursos atuais em curso e futuros cursos (por exemplo, para pessoas que trabalham e desejam fazer um curso este ambiente é aliciante). No início, uma desvantagem era a falta de conteúdos; hoje em dia podem lá colocar-se documentos, ficheiros de áudio, vídeo, vídeo-conferência; etc. Até quem está a decidir sobre um curso/área a escolher para estudo ou negócio, pode simulálo para decidir melhor. Outra fonte de inovação e flexibilidade é a realidade aumentada 
(augmented reality) que pode criar interações mais autênticas dos jogos e dos mundos virtuais com o ambiente que nos rodeia. Baseia-se na projeção e sobreposição de imagens ou objetos virtuais no meio envolvente, dando a ilusão que esse ambiente virtual está à nossa volta. Esta capacidade tornou-se ainda mais acessível através dos smartphones e tablets. Por exemplo, a realidade aumentada pode ser usada em aplicativos que operam em conjunto com câmaras, GPS, entre outros dispositivos, servindo como indicadores de localização daquilo que o cliente deseja encontrar junto com o que existe à sua volta em termos de serviços e novidades relacionadas. Na educação, tais aplicativos podem ser integrados com os jogos de aprendizagem no sentido dos alunos poderem jogar de outras formas ou em outros locais virtualizados.

\section{Conclusão}

Esta investigação pretende compreender a adesão e potencial dos jogos de simulação na educação superior incluindo vantagens, desvantagens e tendências (exemplificando com o jogo Cesim SimBrand). Apesar do limitado uso de jogos sérios em Portugal, os estudantes têm revelado uma atitude positiva devido ao seu envolvimento mais ativo na aprendizagem. Embora nenhum participante (no questionário usado) tivesse experiência anterior com jogos/simulações no ensino, a maioria referiu a importância da aprendizagem baseada-em-jogos. Segundo a maioria dos respondentes, o jogo em estudo permite uma competição realista. Esta foi uma característica-chave apontada, por permitir um melhor entendimento das principais áreas de negócio envolvidas. Foram referidas outras vantagens tais como: mudanças nos procedimentos de trabalho (novos cenários de negócio), experiências "reais" daí resultantes e compreensão dos tópicos da matéria. A experiência geral foi positiva e os estudantes expressaram a sua vontade de ter aprendizagem baseada-em-jogos (GBL) em outras disciplinas. Assim, tendo por base o mesmo questionário, um futuro trabalho poderá comparar a adesão e aplicação deste (ou de outros jogos) em outras disciplinas/cursos.

Estando os estudantes cada vez mais envolvidos com diversas tecnologias (dispositivos móveis, redes sociais, etc.) e face aos desafios dos mundos virtuais e da realidade aumentada, os ambientes tradicionais de ensino tornam-se menos flexíveis e atrativos (Santos et al., 2013). Uma abordagem mista (blended approach) - tradicional junto com novas práticas-podeminimizar certos problemas oulacunas educativas(Duarte\&Martins, 2013). Além de vantagens de aprendizagem, tal abordagem pode ser eficaz em termos de custos (cost effective). Contudo, isso requer planeamento apropriado e estruturas sociais e tecnológicas que possibilitem a sua integração nos programas das disciplinas/cursos. Face aos atuais desafios e tendências, este trabalho reflete a necessidade de repensar materiais e atividades curriculares (disciplinas, cursos, etc.) incluindo estágios e projetos com o exterior. Uma questão a investigar posteriormente poderá incidir no potencial de tais iniciativas para o empreendedorismo e empregabilidade. 


\section{Referências}

Alaranta, M. (2006). Combining theory-testing and theory-building analyses of case study data. Disponível em http://aisel.aisnet.org/ecis2006/175/ (acedido em 29 de Abril de 2014).

Aldrich, C. (2009). The Complete Guide to Simulations and Serious Games: How the Most Valuable Content Will be Created in the Age Beyond Gutenberg to Google. San Francisco: Pfeiffer.

Anderson, J.R. (2006). On cooperative and competitive learning in the management classroom. Mountain Plains Journal of Business and Economics-Pedagogy, 7, 1-10.

Burns, R. (2000). Introduction to research methods. London: Sage Publishers.

Castells, M., Fernandez-Ardevol, M., Qiu, J. \& Sey, A. (2009). Mobile Communication and Society: A Global perspective. Cambridge, MA: MIT Press.

Cesim (2013). Cesim SimBrand. Disponível em http://www.cesim.com/simulations/ cesim-simbrand-marketing-management-simulation-game/ (acedido em o1 de Maio de 2014).

Connolly, T., Boyle, E., MacArthur, E., Hainey, T. \& Boyle, J. (2012). A systematic literature review of empirical evidence on computer games and serious games, Computers \& Education, 59(2), 661-686. doi:10.1016/j.compedu.2012.03.004

Duarte, D. \& Martins, P. (2013). A maturity model for higher education institutions, Journal of Spatial and Organizational Dynamics, 1(1), 25-45.

Eastwood, J. \& Sadler, T. (2013). Teacher's implementation of game-based biotechnology curriculum, Computers \& Education, 66(7), 11-24. doi:10.1016/j. compedu.2013.02.003

CMR (2014). Digital games go online. Centre for Media Research. Disponível em http://www.oecd.org/sti/ieconomy/34078416.pdf (acedido em 11 de Novembro de 2015).

Findling, J. C. (2008). Integration of Game-Based Learning into a social studies curriculum model to improve student performance in the Ohio social studies standarts. Graduate College of Bowling Green State University.

Gall, M., Gall, J. \& Borg, W. (2003). Educational research an introduction. Boston MA: Allyn and Bacon.

Geanellos, R. (2000). Exploring Ricoeur's hermeneutic theory of interpretation as a method of analysing research texts, Nursing Inquiry, 7(2), 112-119.

Golding, C. (2009). Integrating the disciplines: Successful interdisciplinary subjects. Melbourne: The Universirty of Melbourne.

Guillén-Nieto, V. \& Aleson-Carbonell, M. (2012). Serious games and learning effectiveness: The case of it's a deal!, Computers \& Education, 58(1), 435-448. doi:10.1016/j.compedu.2011.07.015 
Hoffman, R. \& Casnocha, B. (2013). The Start-up of You: Adapt to the Future, Invest in Yourself, and Transform Your Caree. New York: Crown Business.

Horizon Report (2014), The NMC Horizon Report: 2014 Higher Education Edition. Disponível em http://www.nmc.org/pdf/2014-nmc-horizon-report-he-EN.pdf (acedido em 27 de Julho de 2015).

Ixmatlahua, S., Raygoza, R., Romero, O., Uribe, F. \& Vargas, E. (2015). Metrópoli Digital: Una plataforma web para la inclusión integral de las PyMES, Sociedad y Gobierno en el uso de las tecnologías de la información en la región de las Altas Montañas del estado de Veracruz, México. Revista Ibérica de Sistemas e Tecnologias de Informação, E3, 43-54. doi:10.17013/risti.e3.43-54

Johnston, H. \& Whitehead, A. (2006), Distinguishing Games, Serious Games, and Training Simulators on the Basis of Intent. Ontario, Canada: School of Information Technology Carleton University.

Kardynal, J. (2009). The potential of serious games for teaching construction technologies: a case study. Disponível em http://library.usask.ca/theses/available/ etd-04082009-194817 (acedido em 20 de Março de 2013).

Kikot, T., Fernandes, S., Magalhães, R. \& Costa, G. (2013), Implementation of business simulation games as learning tools in higher education: An example from University of Algarve, In G. Costa et al. (Eds.), CEPE 2013 Proceedings (Pen: paper 25). Autónoma University, Lisbon.

Lifelong Learning Programme (2012). Success stories - Compilation of game-based learning initiatives in adults' education. Disponível em http://www.p4i-project.eu/ admin-gestion/resultados/files/Success\%20stories-compilation\%20of\%20gamebased\%2olearning\%2oinitiatives\%20in\%20adults\%2oeducation.pdf (acedido em 30 de Abril de 2014).

Martin, S., Diaz, G., Sancristobal, E., Gil, R., Castro, M. \& Peire, J. (2011). New technology trends in education: Seven years of forecast and convergence, Computers \& Education, 57(3), 1893-1906. doi:10.1016/j.compedu.2011.04.003

McClarty, K. L., Orr, A., Frey, P. M., Dolan, R., Vassileva, V. \& McVay, A. (2012). A Literature Review of Gaming in Education. Research Report, Pearson.

Papastergiou, M. (2009). Digital game-based learning in high school computer education: Impact on educational effectiveness and student motivation, Computers \& Education, 52(1), 1-12. doi:10.1016/j.compedu.2008.06.004

Radnitzky, G. (1970). Contemporary schools of metascience. 2nd Ed. Gothenburg: Akademiforlaget.

Salas, E., Wildman, J. \& Piccolo, R.F. (2009). Using simulation based training to enhance management education, Academy of Management Learning and Education, 8(4), 559-573. 
Santos, J.; Gonçalves, G. \& Gomes, A. (2013). Organizational culture and subjective and work well-being. The case of employees of Portuguese universities, Journal of Spatial and Organizational Dynamics, 1(3), 153-161.

Siewiorek, A., Saarinen, E., Lainema, T. \& Lehtinen, E. (2012). Learning leadership skills in a simulated business environment, Computers \& Education, 58(1), 121-135. doi:10.1016/j.compedu.2011.08.016

Sizo, A., Lino, A. \& Favero, E. (2010). Uma proposta de arquitetura de software para construção e integração de ambientes virtuais de aprendizagem. Revista Ibérica de Sistemas e Tecnologias de Informação, 6, 17-30.

Sorensen, M. (2011). Evaluating hotel simulation games effectiveness on higher education performance within service and hospitality. Unpublished master dissertation, Copenhagen Business School.

Virvou, M. \& Alepis, E. (2005), Mobile educational features in authoring tools for personalized tutoring, Computers \& Education, 44, 53-68. doi: 10.1016/j. compedu.2003.12.020

Wall, J. \& Ahmed, V. (2008). Use of a simulation game in delivery blended lifelong learning in the construction industry- Opportunities and challenges, Computers \& Education, 50(4), 1383-1393. doi:10.1016/j.compedu.2006.12.012

Yang, Y.T. \& Wu, W.C. (2012), Digital storytelling for enhancing student academic achievement, critical thinking, and learning motivation: A year-long experimental study, Computers \& Education, 59, 339-352. doi:10.1016/j.compedu.2011.12.012

Zantow, K. \& Knowlton, D. (2005). More than fun and games: Reconsidering the virtues of strategic management simulations, Academy of Management Learning and Education, 4(4), 451-458. doi:10.5465/AMLE.2005.19086786 